\title{
BMJ Open Impact of a cancer diagnosis on the income of adult cancer survivors: a scoping review protocol
}

\author{
Shiraz El Adam (D) , ,2 Colene Bentley, ${ }^{1,2}$ Lisa McQuarrie, ${ }^{1,2}$ Paulos Teckle, ${ }^{1,2,3}$ \\ Stuart Peacock ${ }^{1,2,4}$
}

To cite: El Adam S, Bentley C, McQuarrie L, et al. Impact of a cancer diagnosis on the income of adult cancer survivors: a scoping review protocol. BMJ Open 2021;11:e047315. doi:10.1136/ bmjopen-2020-047315

- Prepublication history and additional supplemental material for this paper are available online. To view these files, please visit the journal online (http://dx.doi.org/10.1136/ bmjopen-2020-047315)

Received 25 November 2020 Accepted 17 August 2021

Check for updates

(C) Author(s) (or their employer(s)) 2021. Re-use permitted under CC BY-NC. No commercial re-use. See rights and permissions. Published by BMJ.

${ }^{1}$ Cancer Control Research, BC Cancer, Vancouver, British Columbia, Canada

${ }^{2}$ Canadian Centre for Applied Research in Cancer Control, Vancouver, British Columbia, Canada

${ }^{3}$ School of Population and Public Health, University of British Columbia, Vancouver, British Columbia, Canada

${ }^{4}$ Faculty of Health Sciences, Simon Fraser University, Burnaby, British Columbia, Canada

Correspondence to

Shiraz El Adam;

seladam@bccrc.ca

\section{ABSTRACT}

Introduction While the socioeconomic impact of a cancer diagnosis on cancer survivors has gained some attention in the literature, to our knowledge, a review of the evidence on changes in income due to cancer has yet to be undertaken. In this paper, we describe a scoping review protocol to review the evidence on the effect of a cancer diagnosis on the income of individuals diagnosed with cancer during adulthood ( $\geq 18$ years). The purpose is to summarise existing evidence, identify gaps in current research and highlight priority areas for future research. Methods and analysis This study will follow the methodological framework for conducting scoping reviews by the Joanna Briggs Institute In collaboration with a health science librarian, we developed a search strategy to be performed in Ovid MEDLINE, PsycINFO, Cumulative Index to Nursing and Allied Health Literature, EMBASE, Econ-Literature and Evidence-Based Medicine Reviews. This scoping review will search the scientific literature published in English from 1 January 2000 to 31 December 2020. Studies that measured the impact of cancer on income of adults will be eligible for inclusion. Studies exclusively focused on employment outcomes (eg, return to work, unemployment, productivity loss), financial expenditures, childhood cancer survivors and/or the caregivers of cancer survivors will be excluded. Three independent reviewers will conduct screening and extract data. Descriptive information will be reported following the Preferred Reporting Items for Systematic Reviews and Meta-Analyses (PRISMA) extension for Scoping Reviews.

Ethics and dissemination This scoping review will analyse data from publicly available materials and thus does not require ethics approval. Results from this review will be disseminated through a peer-reviewed publication and/or conference presentation with the potential to identify gaps in the literature, suggest strategies for standardised terminology and provide directions for future research.

\section{INTRODUCTION}

In 2018, the World Health Organization estimated the number of cancer cases will increase from 18.1 million in 2018 to 29.5 million $(+63.4 \%)$ in $2040 .{ }^{1}$ Similarly, the number of people surviving cancer beyond 5 years since diagnosis is also increasing. A population-based study on cancer survival

\section{Strengths and limitations of this study}

- This scoping review will be the first review to summarise results of studies looking specifically at changes in all sources of income of individuals diagnosed with cancer during adulthood.

- The identification and summary of data will involve a systematic search of six online databases over the last 20 years.

- This study follows a rigorous methodology based on the latest guidelines for scoping reviews updated by the Joanna Briggs Institute (2020), and the Preferred Reporting Items for Systematic Reviews and MetaAnalyses extension for Scoping Reviews (2018).

- A quality assessment of the articles included in the scoping review will not be performed, as this would be beyond the aim of a scoping review.

- Only studies published in English in peer-reviewed journals will be considered eligible for inclusion.

in seven high-income countries showed that between 1995 and 2014, both 1-year and 5-year net survival increased in each country across almost all cancer types, with larger survival improvements for those younger than 75 years at diagnosis. ${ }^{2}$ In this paper, we will refer to cancer survivors as individuals diagnosed with cancer from the time of diagnosis until death. ${ }^{3}$ With the growth and ageing of populations, ${ }^{4}$ and recent advances in cancer control, there are a growing number of individuals living with cancer as a chronic disease and coping with its long term and late effects.

As more people survive a cancer diagnosis, they often live with a wide range of physical and psychological impacts from treatment that can linger for months or years (longterm effects) or may not appear until years after treatment ends (late effects). ${ }^{5}$ Examples of long-term effects that emerge during or after cancer treatment and persist in a chronic long-term manner include: neuropathies, with related weakness, numbness or pain; fatigue; cognitive or sexual difficulties; elevated anxiety or depression; and impaired 
cardiac function. ${ }^{56}$ In contrast, late effects may develop as a result of treatment on organ systems or the overall psychological process, such as musculoskeletal complications or late-onset stamina deficits related to cardiovascular complications or hypothyroidism. ${ }^{5}$

In addition to the emotional and physical burdens that cancer survivors have to bear, mounting treatment costs and productivity losses due to reduced employment can further exacerbate the burden of cancer at individual and societal levels. ${ }^{6-8}$ From a societal perspective, improved survival outcomes have enabled cancer survivors to return to work and participate in social and economic life, but often at a reduced capacity. A systematic review on occupationally active cancer survivors found that their work abilities were negatively influenced by cognitive limitations, fatigue, coping issues, depression and anxiety, in addition to physical limitations, such as difficulties with lifting and treatment-induced menopausal symptoms. ${ }^{9}$ When measuring the societal burden of cancer in Europe, for instance, the costs of lost productivity due to cancerrelated premature mortality, in those between the ages of 15 and 64 years, represented a loss of $€ 75$ billion to European economies in $2008(0.58 \%$ of Gross Domestic Product)..$^{10}$

At an individual level, reduced labour force activity can result in income loss and financial distress. Studies have shown that cancer-related financial stress has considerable negative effects on the quality of life of individuals with cancer, ${ }^{11}$ leading in some cases to higher risks of mortality. ${ }^{12}$ A systematic review of 45 studies on financial hardship in the USA found financial distress in 47\%-49\% of cancer survivors, and that $4 \%-45 \%$ of survivors did not adhere to recommended prescription medication due to cost. ${ }^{13}$ In low-income minority populations, financial strain may further contribute to difficulties retaining employment or returning to work, ${ }^{67}$ as well as to challenges accessing supportive resources, such as hospitalbased or community-based cancer support groups. ${ }^{14}$ A study looking at extreme financial distress found that patients with cancer who declared bankruptcy had a $79 \%$ greater mortality risk than those who had not. ${ }^{12}$

The relationship between cancer-related financial stress and increased mortality is the result of various complex pathways, three of which have been explained through: poorer subjective well-being; impaired health-related quality of life (HRQOL) and subpar quality of care. ${ }^{16}$ Poorer subjective well-being-defined as 'valuations that people make regarding their lives, the events happening to them, their bodies and minds, and the circumstances in which they live ${ }^{17}$ — can be experienced by cancer survivors as a result of cutting back on leisure activities, spending less on food and clothing, and working longer hours ${ }^{18}$ to meet the high out-of-pocket costs of treatment. This, in turn, might impact health outcomes including survival. ${ }^{1920}$ A study looking at cancer survivors' HRQOL found that greater financial hardship was the strongest independent predictor of reporting worse HRQOL. ${ }^{21}$ Similarly, among 1000 patients with lung or colorectal cancer, high financial burden was associated with worse self-reported HRQOL. ${ }^{22}$ Finally, in terms of quality of care, studies suggest that non-adherence to potentially life-saving drugs can be induced from relatively small reductions in cancer survivors' budget-less than a US\$100 per month ${ }^{162324}$. For instance, among women receiving adjuvant hormonal breast cancer therapy greater monthly copayments-ranging from US\$30 to US $\$ 90$ for those drugs-were associated with increased odds of non-compliance. ${ }^{23}$

Considered together, the accumulation of these adverse effects has a considerable socioeconomic impact on individuals surviving cancer and on society in general.

\section{Study rationale}

While the socioeconomic impact of a cancer diagnosis on cancer survivors has attracted some attention in the literature, to our knowledge a review summarising the evidence on changes in different sources of income of individuals diagnosed with cancer during adulthood $(\geq 18$ years) has yet to be undertaken.

Our scoping review will summarise the existing literature on the effect of a cancer diagnosis on all sources of income of adult cancer survivors. We will refer to adult cancer survivors as individuals diagnosed with cancer during adulthood (ie, 18 years and older) and only report results that fall within this study population. We have chosen to exclude individuals diagnosed with cancer during childhood (ie, from birth to just before the individual's 18th birthday) from our study population, due to the age-dependent outcomes being studied and the existence of a previous review of the effect of a childhood cancer diagnosis on household income. ${ }^{25}$ However, we will not include an age limit in our search to avoid missing studies that include adolescents and young adults (AYAs) due to age overlap. AYA is defined variously as 15-39 years in the USA (National Cancer Institute definition ${ }^{26}$ ) and 15-24 years in Europe. ${ }^{27}$ Therefore, despite including a broader age group category in our search term, we will only report results of studies restricted to the 18 years and older population. For studies on AYAs, we will only include and report those that have stratified their results by age, to 18 years and over, if applicable. This population is of direct interest to us, as it constitutes a large part of the working age population. One recent study that focused on understanding and measuring the financial impact of cancer on AYAs highlighted this age group's particular vulnerability to treatment-related financial distress and its clinical manifestations (interrupted work and income loss, debt accumulation, treatment non-adherence, avoidance of medical care and social isolation). ${ }^{28}$

Our rationale for choosing all sources of income as our outcome is to summarise the impact of a cancer diagnosis on all individuals regardless of their employment or financial status. A cancer diagnosis is known to impair the employment and/or the working conditions of survivors, ${ }^{9}$ and potentially their income source. Looking at employment income exclusively would introduce a bias in our 
results towards cancer survivors who remain employed postcancer diagnosis. By looking at all sources of income, such as unemployment income, income from savings or physical assets and household income, we eliminate this bias, and provide a more comprehensive understanding of the overall effect of a cancer diagnosis on income.

Over the last two decades, researchers have employed various outcomes to measure the financial impact of a cancer diagnosis on individuals, such as financial burden, ${ }^{29}$ financial distress ${ }^{30}$ and most recently financial toxicity (FT). ${ }^{31}$ FT has been defined in oncology research as the financial distress or hardship that may be a side effect of cancer treatment. ${ }^{16} 3233$ Conceptually, the term FT has been described as the unintended objective financial burden resulting from direct out-ofpocket medical expenses related to cancer treatment and subjective financial distress resulting from the accumulation of cancer-related expenditures and reduction in wealth combined with the anxiety and discomfort experienced by the patient over their cancer experience. ${ }^{31}$ A recent systematic review on the FT among individuals with cancer identified a variety of outcome measures (70 outcomes of FT) and called for further research in order to standardise outcomes as well as income losses. ${ }^{34}$ While these outcomes are particularly concerned with drugrelated and treatment-related financial burden, they often overlook the overall impact of a cancer diagnosis on the income of adults. Given the variety of definitions in the literature, our research team has chosen to follow the economic definitions: income will refer to 'the flow deriving from a stock of wealth', where wealth includes physical wealth, financial wealth and human capital, resulting from past investments in education and training or natural talent. ${ }^{35}$

To identify any existing reviews on the topic of income of adult cancer survivors, we conducted a preliminary literature search for all articles published between 2000 and May 2020 in three databases: Ovid Medline, PROSPERO and COCHRANE. Depending on the database searched, we combined search terms to identify our study population (adults), intervention (cancer, malignancy or neoplasm) and our outcomes (income or financial or economic).

Our preliminary search yielded a few reviews in addition to numerous studies investigating the financial hardship from a cancer diagnosis. Two systematic reviews focused on childhood cancer survivors, ${ }^{25} 36$ one systematic review focused on Hodgkin lymphoma, ${ }^{37}$ others looked at $\mathrm{FT}^{34}$ and financial hardship in the $\mathrm{USA}^{13}$ and several reviews including a meta-review looking at return-to-work after a cancer diagnosis. ${ }^{8}$

Results from these reviews are noteworthy. The systematic review on Hodgkin lymphoma (39 studies) found that $26 \%-36 \%$ of survivors perceived Hodgkin lymphoma as negatively affecting their socioeconomic status. ${ }^{37}$ This review further pointed to the challenge of comparability due to the lack of standardised reporting methods. The systematic review on childhood cancer survivors (10 studies) and the effect of childhood cancer on income found that survivors of various childhood cancers (particularly Central Nervous System tumours) had a lower income than the general population or a sibling comparison group. ${ }^{25}$ The systematic review also finds variations in the definition of source of income.

In an effort to focus our scoping review on gaps in the evidence synthesis literature, we have also excluded parents or caregivers (unless part of the household) of cancer survivors from our study population, as well as studies specifically focused on occupational outcomes such as employment status, productivity loss (if measured in work hours or days), return-to-work or unmet financial needs. These exclusions are based on our awareness of more rigorous evidence synthesis studies such as systematic reviews that looked at caregivers of patients with cancer ${ }^{38}$ or more specifically parents of patients with cancer, ${ }^{36} \mathrm{FT},{ }^{34}$ financial hardship, ${ }^{13}$ return-to-work after a cancer diagnosis, ${ }^{8}$ as well as work productivity after cancer. $^{39}$

Our scoping review will provide synthesised evidence on the extent of the effect of a cancer diagnosis on the income of adults of different sexes, age groups, cancer types, geographical locations (ie, country of residence) and follow-up periods. Findings from this review will enable us to determine whether a further systematic review is needed, identify vulnerable populations that are at most risk of significant income loss and highlight contextual factors that may have alleviated these effects.

Finally, since several reviews have alluded to the challenges of comparing findings of inconsistent measurements, we will use this scoping review to map out different income outcome definitions and measurements in the literature. We will report the various outcome definitions and measurements used in the literature, including the frequency of use (if common or similar). This will allow us to find common definitions and identify gaps in the literature. We will also map and identify the various working definitions of our study population-adults including AYAs - to help stratify effects by age and to find common definitions. Based on our findings, we will suggest strategies and reporting methods to be used in future studies. This will support future research in finding and working towards consistent and universal definitions that will ease the comparability of results across different settings.

\section{Study objectives}

The aim of this scoping review is to summarise and map the literature on the impact of a cancer diagnosis on the income of adult cancer survivors.

The specific objectives are:

1. To provide an overview of income definitions and measurements used in the current literature.

2. To present review findings in a tabular form displaying study design, methods and key findings including effect, alongside a narrative summary. This will constitute our map of the literature, as per scoping review methodology. 
3. To compare the income effect by cancer type, age, sex and/or gender, and other variables of interest (eg, country of residence, marital status, occupation).

4. To identify gaps in the current literature in terms of data sources, study populations-including cancer type-study design, methodology and outcome definitions and measurements.

\section{METHODS AND ANALYSIS}

A scoping review methodology will be followed to summarise the literature on the effect of a cancer diagnosis on the income of adult cancer survivors. Scoping reviews are typically used to describe the literature on broad research questions. ${ }^{40-42}$

Our methods will follow the most recent methodological framework for conducting a scoping review by the Joanna Briggs Institute (JBI), ${ }^{43}$ which builds on previous frameworks by Arksey \& $\mathrm{O}^{\prime}$ Malley ${ }^{40}$ and Levac et $a t^{42}$. To increase our scoping review's methodological and reporting transparency, we will follow the Preferred Reporting Items for Systematic Reviews and Meta-Analyses (PRISMA) extension for Scoping Reviews, ${ }^{44}$ and ensure the reporting of all the essential items on the checklist (online supplemental material 1).

\section{Research question}

The aim of this scoping review is to summarise the existing evidence on the effect of a cancer diagnosis on the income of adult cancer survivors.

Our scoping review will be guided by the following specific research questions (table 1 ):

\section{Inclusion criteria}

In alignment with the study objective and research questions and following the 'Population-Concept-Context' framework recommended by the JBI, ${ }^{43}$ we developed the following inclusion criteria (table 2) as a basis for study selection and inclusion.

\section{Search strategy}

To develop our search strategy, we followed an iterative approach of piloting and refining the search strategy after evaluating the results with our research team. In collaboration with BC Cancer's health science librarian, our preliminary search strategy was developed using key words and Medical Subject Headings (MeSH) terms from our research question and from similar search strategies in published reviews.

After piloting the preliminary search strategy in Ovid Medline in May 2020, we determined our search strategy was feasible, and will return a significant number of potential studies to include in the review. We will focus our study on adults with a cancer diagnosis, and limit our search to the last 20 years (1 January 2000-31 December 2020). We chose to exclude individuals diagnosed with cancer during childhood from our search without adding an age limit to our search strategy. The rationale for this decision was based on the advice of our librarian and the following issues:

1. Ovid Medline's definition of 'child'-age 0-18yearsand adolescents-age 13-18 years-have an overlap. This overlap may result in excluding studies on AYAs if an age restriction of $>18$ years is added to the search strategy.

2. To ensure that the AYA population-a population of particular interest and a portion of the adult population-is not excluded in our search, we purposely added keywords and MeSH words that refer to this population and combined them with our search.

Our final search strategy can be found in online supplemental material 2. It was developed to perform a scoping review of the scientific literature published in English from 1 January 2000 to 31 December 2020, inclusive, in the following information sources:

1. Electronic databases: Ovid MEDLINE, PsycINFO, Cumulative Index to Nursing and Allied Health

Table 1 Research questions

Research questions

1. How are income outcomes defined and measured in the current literature?

Types of data used

- Outcome definition

Inclusion/exclusion criteria

2. What is the current evidence on the effect of a cancer diagnosis on the income of Number and types of studies adults with cancer?

- Geographical representation

- Magnitude, direction and effect size

- Study population and subgroups

- Study type

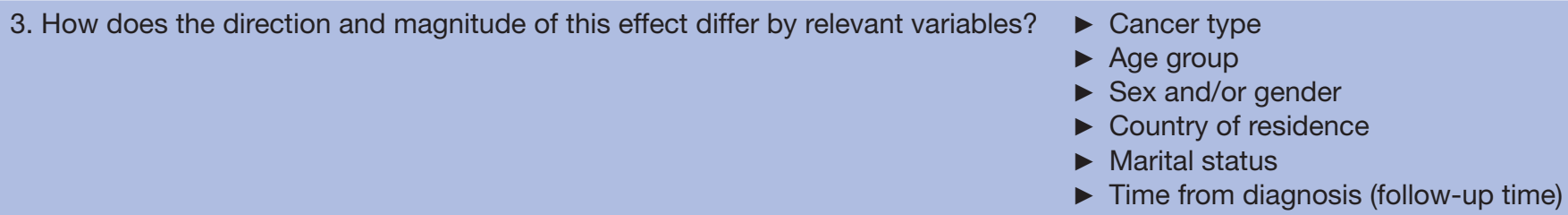

4. Where are the gaps in the current literature and what are the priority areas for future research? 
Table 2 Inclusion and exclusion criteria

\begin{tabular}{|c|c|c|}
\hline & Inclusion & Exclusion \\
\hline Population & $\begin{array}{l}\text { Individuals diagnosed with cancer during adulthood (18 } \\
\text { years or older) regardless of sex/gender, tumour type, } \\
\text { cancer treatment or follow-up time. } \\
\text { Studies on AYAs, or a broader age group, with analyses } \\
\text { stratified by age, including a distinct stratification for } \\
\text { individuals } 18 \text { years and older will be included; however, } \\
\text { only results from individuals diagnosed with cancer } 18 \\
\text { years or older will be reported. }\end{array}$ & $\begin{array}{l}\text { Studies exclusively focused on individuals } \\
\text { diagnosed with cancer during childhood (less than } \\
18 \text { years), or on parents, spouses or caregivers of } \\
\text { cancer survivors will be excluded. } \\
\text { Studies focused on AYAs, without age stratification } \\
\text { for those } 18 \text { years and older will be excluded. }\end{array}$ \\
\hline Concept & $\begin{array}{l}\text { Studies that aim to measure the effect of a cancer } \\
\text { diagnosis on the income* of adult cancer survivors. } \\
\text { Income outcomes will refer to any source of income } \\
\text { including any of the following or similar outcomes: } \\
\text { employment income (individual or household), salary, } \\
\text { earnings, unemployment income, disability income } \\
\text { or disability pension, government assistance, debt, } \\
\text { bankruptcy, income from physical (resource) wealth } \\
\text { (such as assets, properties, vehicles, etc), and financial } \\
\text { wealth (such as shares, government bonds, bank } \\
\text { accounts and savings). }\end{array}$ & $\begin{array}{l}\text { Studies exclusively focused on occupational } \\
\text { outcomes such as employment status, productivity } \\
\text { loss (if measured in work hours or days), return-to- } \\
\text { work or unmet financial needs will be excluded. } \\
\text { Studies exclusively focused on FT, financial burden } \\
\text { or distress measured through cost of care, health } \\
\text { expenditures or out-of-pocket costs, without } \\
\text { measuring income patterns will be excluded. } \\
\text { Economic evaluations or the cost-effectiveness of } \\
\text { selecting one treatment option over another will be } \\
\text { excluded. }\end{array}$ \\
\hline Context & $\begin{array}{l}\text { Studies from any country and/or healthcare system. } \\
\text { Studies published between } 1 \text { January } 2000 \text { and } 31 \\
\text { December 2020, inclusive. }\end{array}$ & $\begin{array}{l}\text { Due to resource and time constraints, only studies } \\
\text { published in English will be included. }\end{array}$ \\
\hline $\begin{array}{l}\text { Types of } \\
\text { studies }\end{array}$ & $\begin{array}{l}\text { Primary quantitative and qualitative research studies } \\
\text { (such as quasi-experimental, before-and-after studies, } \\
\text { prospective and retrospective cohort studies, case- } \\
\text { control studies and analytical cross-sectional studies). } \\
\text { Systematic reviews and/or scoping reviews will be used } \\
\text { to identify relevant primary literature through reference } \\
\text { lists. }\end{array}$ & $\begin{array}{l}\text { Conference proceedings, commentaries, } \\
\text { theoretical, conceptual or normative discussions, } \\
\text { and any non-peer-reviewed literature will be } \\
\text { excluded. }\end{array}$ \\
\hline
\end{tabular}

*Income outcomes will refer to outcomes measuring 'the flow deriving from a stock of wealth', where wealth includes physical wealth, financial wealth and human capital-resulting from past investments in education and training or natural talent. ${ }^{35}$

AYAs, adolescents and young adults; FT, financial toxicity.

Literature, EMBASE, Econ-Literature and EvidenceBased Medicine Reviews (full list in online supplemental material 2).

2. Reference lists: We will search through the bibliographies of studies identified in the database searchesspecifically systematic reviews and traditional literature reviews-to ensure that all relevant studies have been included in the scoping exercise.

\section{Evidence selection}

After running the searches in the six electronic databases mentioned above, we will import all records to Endnote for bibliographic management and deduplication. Using the eligibility criteria (table 2), a simple screening template was developed (online supplemental material 3) to aid with the screening of titles and abstracts. During this phase, all records combined will be distributed between three independent reviewers. Reviewers will be asked to screen the titles and abstracts of these records into three separate folders: 'relevant studies', 'irrelevant studies' and 'potentially relevant studies that need a fulltext assessment'. Consequently, two reviewers will screen the same full-text articles in the 'potentially relevant' pile for inclusion, independently. Inter-rater discrepancies will be resolved by a third reviewer and discussion with the research team.

\section{Data extraction}

We tailored the data extraction template by $\mathrm{JBI}^{43}$ to align with our overall objective and research questions (online supplemental material 4).The charting form will be updated in an iterative process as more variables are discovered in the included studies. Two reviewers will pilot the data extraction template by independently extracting data from the first 10 included studies, and meet to determine whether the form is suitable for addressing the scoping review research question. After piloting the template, updates to the data extraction form will be made if necessary and based on consensus of the research team. Similar to the study selection approach above, inter-rater discrepancies in the data extraction will be resolved by a third reviewer and discussion with the research team. This iterative approach is encouraged in the guidelines for a scoping review. 404546

Data will initially be charted using the following headings: 
1. Author(s) and journal.

2. Year of publication.

3. Country where the study was conducted.

4. Aims/purpose.

5. Study population and sample size (including age group definitions if applicable).

6. Methodology (study design, period, data sources and recruitment).

7. Cancer type and duration/follow-up time.

8. Outcomes studied (including outcome definition and measurement).

9. Subgroups and/or covariates considered.

10. Main findings.

\section{Data analysis and presentation of the results}

We will summarise the results from this review through a narrative synthesis in line with the recommendations set out in the PRISMA extension for Scoping Reviews ${ }^{44}$ (online supplemental material 1). After identifying the breadth of the income outcomes of adult cancer survivors in the literature, we will report the results by each outcome, cancer type, sex and age group (where applicable). Variations in these subgroups and other considered subgroups will be further explained in the narrative synthesis. We will present our results through the following:

1. Table 1: A frequency table on the subquestions in table 1 (simple frequency counts based on the data abstracted above) will be used to describe our findings.

2. Table 2: We will chart the data from all eligible studies to describe the different methods used, country of study, population characteristics, outcomes, outcome definition and measurement, subgroups and main findings. This will allow us to visualise gaps in the literature in terms of methods, population and context.

Finally, we will summarise the evidence in relation to the purpose of the review, and discuss results in terms of the different study contexts (such as key features of the health system-eg, public/private finance, public/ private delivery; type social security/welfare system; low/ middle/high income country) and consistency of measurements and definitions (particularly for age group and outcome definitions). Based on our findings, we will suggest strategies for standardising reporting methods in future studies and discuss the overall implications of the results for research and policy.

\section{Patient and public involvement}

No patient involved.

\section{Ethics and dissemination}

Since this scoping review is based on reviewing and collecting data from publicly available materials, it does not require ethics approval.

To the best of the research team's knowledge, this is the first review to summarise the evidence on the effect of cancer diagnosis on the income of adult cancer survivors.

We will disseminate the results of this review through a peer-reviewed publication and/or conference presentation. By summarising the evidence on this topic and identifying gaps in the literature, this scoping review can provide direction for future primary research and systematic reviews or meta-analyses.

\section{Twitter Shiraz El Adam @ShirazEIAdam1}

Acknowledgements We would like to acknowledge Elena Popova (reference Librarian, BC Cancer Library) for her assistance in developing our comprehensive search strategy, and Claire de Oliveira, Beverley Essue and Helen McTaggart-Cowan for reviewing and providing feedback on the protocol.

Contributors SEA developed the research questions, initial search strategy, methods and drafted the protocol. CB contributed to the development of the methods, and to substantial editing of the protocol. LM, PT and SP contributed to the screening of studies in the preliminary search. All authors contributed to the discussion and preparation of screening templates. PT and SP provided subjectmatter expert feedback throughout the preparation of the protocol. SP supervised the preparation of the protocol and critically reviewed the manuscript. All authors approved the final manuscript.

Funding This work was supported by funding from the Canadian Partnership Against Cancer (CPAC) and the Canadian Centre for Applied Research in Cancer Control (ARCC) (Grant \# 2020-706936).

Competing interests None declared.

Patient consent for publication Not required.

Provenance and peer review Not commissioned; externally peer reviewed.

Supplemental material This content has been supplied by the author(s). It has not been vetted by BMJ Publishing Group Limited (BMJ) and may not have been peer-reviewed. Any opinions or recommendations discussed are solely those of the author(s) and are not endorsed by BMJ. BMJ disclaims all liability and responsibility arising from any reliance placed on the content. Where the content includes any translated material, BMJ does not warrant the accuracy and reliability of the translations (including but not limited to local regulations, clinical guidelines, terminology, drug names and drug dosages), and is not responsible for any error and/or omissions arising from translation and adaptation or otherwise.

Open access This is an open access article distributed in accordance with the Creative Commons Attribution Non Commercial (CC BY-NC 4.0) license, which permits others to distribute, remix, adapt, build upon this work non-commercially, and license their derivative works on different terms, provided the original work is properly cited, appropriate credit is given, any changes made indicated, and the use is non-commercial. See: http://creativecommons.org/licenses/by-nc/4.0/.

ORCID iD

Shiraz El Adam http://orcid.org/0000-0002-8036-2495

\section{REFERENCES}

1 IARC. World Health Organization International Agency for Research on Cancer (IARC) - Global Cancer Observatory (GLOBOCAN). Geneva: World Health Organization, 2018.

2 Arnold M, Rutherford MJ, Bardot A, et al. Progress in cancer survival, mortality, and incidence in seven high-income countries 1995-2014 (ICBP SURVMARK-2): a population-based study. Lancet Oncol 2019;20:1493-505

3 Denlinger CS, Carlson RW, Are M, et al. Survivorship: introduction and definition. J Natl Compr Canc Netw 2014;12:34-45.

4 Popat K, McQueen K, Feeley TW. The global burden of cancer. Best Pract Res Clin Anaesthesiol 2013;27:399-408.

5 Stein KD, Syrjala KL, Andrykowski MA. Physical and psychological long-term and late effects of cancer. Cancer 2008;112:2577-92.

6 De Anda RM. Employment hardship among Mexican-Origin women. Hisp J Behav Sci 2005;27:43-59.

7 Taskila-Brandt T, Martikainen R, Virtanen SV, et al. The impact of education and occupation on the employment status of cancer survivors. Eur J Cancer 2004;40:2488-93.

8 Butow P, Laidsaar-Powell R, Konings S, et al. Return to work after a cancer diagnosis: a meta-review of reviews and a meta-synthesis of recent qualitative studies. J Cancer Surviv 2020;14:114-34.

9 Duijts SFA, van Egmond MP, Spelten E, et al. Physical and psychosocial problems in cancer survivors beyond return to work: a systematic review. Psychooncology 2014;23:481-92. 
10 Hanly P, Soerjomataram I, Sharp L. Measuring the societal burden of cancer: the cost of lost productivity due to premature cancer-related mortality in Europe. Int J Cancer 2015;136:E136-45.

11 Kale HP, Carroll NV. Self-Reported financial burden of cancer care and its effect on physical and mental health-related quality of life among US cancer survivors. Cancer 2016;122:283-9.

12 Ramsey SD, Bansal A, Fedorenko CR, et al. Financial insolvency as a risk factor for early mortality among patients with cancer. $J$ Clin Oncol 2016;34:980-6.

13 Altice CK, Banegas MP, Tucker-Seeley RD, et al. Financial hardships experienced by cancer survivors: a systematic review. J Natl Cancer Inst 2017;109. doi:10.1093/jnci/djw205. [Epub ahead of print: 2010 2016].

14 Ell K, Xie B, Wells A, et al. Economic stress among lowincome women with cancer: effects on quality of life. Cancer 2008;112:616-25.

15 Guidry JJ, Aday LA, Zhang D, et al. Cost considerations as potential barriers to cancer treatment. Cancer Pract 1998;6:182-7.

16 Zafar SY. Financial toxicity of cancer care: it's time to intervene. J Natl Cancer Inst 2016;108. doi:10.1093/jnci/djv370. [Epub ahead of print: 1112 2015]

17 Camfield L, Skevington SM. On subjective well-being and quality of life. J Health Psychol 2008;13:764-75.

18 Zafar SY, Peppercorn JM, Schrag D, et al. The financial toxicity of cancer treatment: a pilot study assessing out-of-pocket expenses and the insured cancer patient's experience. Oncologist 2013;18:381-90.

19 Diener E, Chan MY. Happy people live longer: subjective well-being contributes to health and longevity. Appl Psychol 2011;3:1-43.

20 Lamers SMA, Bolier L, Westerhof GJ, et al. The impact of emotional well-being on long-term recovery and survival in physical illness: a meta-analysis. J Behav Med 2012;35:538-47.

21 Fenn KM, Evans SB, McCorkle R, et al. Impact of financial burden of cancer on survivors' quality of life. J Oncol Pract 2014;10:332-8.

22 Zafar SY, McNeil RB, Thomas CM, et al. Population-based assessment of cancer survivors' financial burden and quality of life: a prospective cohort study. J Oncol Pract 2015;11:145-50.

23 Neugut Al, Subar M, Wilde ET, et al. Association between prescription co-payment amount and compliance with adjuvant hormonal therapy in women with early-stage breast cancer. J Clin Oncol 2011;29:2534-42.

24 Dusetzina SB, Winn AN, Abel GA, et al. Cost sharing and adherence to tyrosine kinase inhibitors for patients with chronic myeloid leukemia. J Clin Oncol 2014;32:306-11.

25 Frederiksen LE, Mader L, Feychting M, et al. Surviving childhood cancer: a systematic review of studies on risk and determinants of adverse socioeconomic outcomes. Int J Cancer 2019;144:1796-823.

26 Smith AW, Keegan T, Hamilton A, et al. Understanding care and outcomes in adolescents and young adults with cancer: a review of the AYA hope study. Pediatr Blood Cancer 2019;66:e27486.

27 Gatta G, Zigon G, Capocaccia R, et al. Survival of European children and young adults with cancer diagnosed 1995-2002. Eur J Cancer 2009;45:992-1005.
28 Salsman JM, Bingen K, Barr RD, et al. Understanding, measuring, and addressing the financial impact of cancer on adolescents and young adults. Pediatr Blood Cancer 2019;66:e27660.

29 Delgado-Guay M, Ferrer J, Rieber AG, et al. Financial distress and its associations with physical and emotional symptoms and quality of life among advanced cancer patients. Oncologist 2015;20:1092-8.

30 Workman P, Draetta GF, Schellens JHM, et al. How much longer will we put up with $\$ 100,000$ cancer drugs? Cell 2017;168:579-83.

31 Carrera PM, Kantarjian HM, Blinder VS. The financial burden and distress of patients with cancer: understanding and stepping-up action on the financial toxicity of cancer treatment. CA Cancer J Clin 2018;68:153-65.

32 de Souza JA, Wong Y-N. Financial distress in cancer patients. J Med Person 2013;11:73-7.

33 Tucker-Seeley RD, Yabroff KR. Minimizing the "financial toxicity" associated with cancer care: advancing the research agenda. J Natl Cancer Inst 2016;108:djv410.

34 Gordon LG, Merollini KMD, Lowe A, et al. A systematic review of financial toxicity among cancer survivors: we can't pay the co-pay. Patient 2017;10:295-309.

35 Barr N. The economics of the welfare state third edition. Education. Oxford: Oxford University Press, 1993.

36 Roser K, Erdmann F, Michel G, et al. The impact of childhood cancer on parents' socio-economic situation systematic review. Psychooncology 2019;28:1207-26.

37 Pálmarsdóttir R, Kiesbye Øvlisen A, Severinsen MT, et al. Socioeconomic impact of Hodgkin lymphoma in adult patients: a systematic literature review. Leuk Lymphoma 2019;60:3116-31.

38 Kusi G, Boamah Mensah AB, Boamah Mensah K, et al. The experiences of family caregivers living with breast cancer patients in low-and middle-income countries: a systematic review. Syst Rev 2020;9:165

39 Kamal KM, Covvey JR, Dashputre A, et al. A systematic review of the effect of cancer treatment on work productivity of patients and caregivers. J Manag Care Spec Pharm 2017;23:136-62.

40 Arksey H, O'Malley L. Scoping studies: towards a methodological framework. Int J Soc Res Methodol 2005;8:19-32.

41 Colquhoun HL, Levac D, O'Brien KK, et al. Scoping reviews: time for clarity in definition, methods, and reporting. J Clin Epidemiol 2014;67:1291-4.

42 Levac D, Colquhoun H, O'Brien KK. Scoping studies: advancing the methodology. Implementation Sci 2010;5:1-9.

43 Peters MDJ, Godfrey CM, Mclnerney P. Chapter 11: scoping reviews (2020 version). In: Joanna Briggs Institute reviewer's manual. Adelaide, Australia: JBI, 2020.

44 Tricco AC, Lillie E, Zarin W, et al. PRISMA extension for scoping reviews (PRISMA-ScR): checklist and explanation. Ann Intern Med 2018;169:467-73.

45 Armstrong R, Hall BJ, Doyle J, et al. 'Scoping the scope' of a cochrane review. J Public Health 2011;33:147-50.

46 Valaitis R, Martin-Misener R, Wong ST, et al. Methods, strategies and technologies used to conduct a scoping literature review of collaboration between primary care and public health. Prim Health Care Res Dev 2012;13:219-36. 